\title{
RESUMEN
}

El siguiente trabajo indica los resultados de la aplicación de 350 encuestas on line en la región metropolitana de Santiago de Chile entre el 20 de marzo y el 20 de abril del año 2019. Dicha encuesta fue construida y adaptada en base en las escalas desarrolladas por (Zeithaml, Parasuraman y Malhotra; 2000), que se aplicaron con el objetivo de recopilar y analizar los resultados obtenidos a partir de las respuestas de los consumidores que realizaron compras a través de Amazon. Se aplicaron las dos escalas propuestas por Ios autores; E-S-QUAL o escala principal en donde las dimensiones evaluadas fueron: eficiencia, disponibilidad del sistema, cumplimiento, privacidad y la escala E-RecS-QUAL que busca medir la recuperación o el restablecimiento cuando un cliente tiene problemas con el servicio, para esta segunda escala se midieron las dimensiones: valor percibido, post venta y lealtad. Esto nos permitió visualizar la percepción de los consumidores producto del servicio entregado por esta compañía y de esa forma poder visualizar oportunidades de mejora y crecimiento para los e-tailers en el contexto latinoamericano.

Palabras Clave: e-tail, e-s-qual, e-rec-qual, calidad de servicio on line

\section{APLICACIÓN DE ESCALAS E-S-QUAL Y E-REC-QUAL PARA EVALUAR LA CALIDAD DEL SERVICIO DEL E-TAIL AMAZON EN CHILE \\ Luis Leyton-Johns ${ }_{1}$ / Ricardo Ahumada Castillo ${ }_{2}$ / Joan Luis Mora Díaz}

\author{
Fecha de recepción: 30 de julio de 2020 \\ Fecha de aceptación: 24 de octubre de 2020
}

\section{DOI: https://doi.org/10.22370/riace.2020.9.1.2599}

1 Corresponding Author. Universidad Andres Bello, Santiago, Chile, leyton.johns@gmail.com, ORCID ID: 0000-0002-4462-403X.

2 Universidad Tecnológica de Chile, Talca, Chile, ricardo.ahumada.castillo@gmail.com, ORCID ID: 0000-0003-3558-5232.

3 Universidad Católica Cecilio Acosta, joanluismora@gmail.com, ORCID ID: 0000-0002-2943-1205. 


\title{
APPLICATION OF E-S-QUAL AND E-REC-QUAL SCALES TO EVALUATE THE EUALITY OF THE AMAZON E-TAIL SERVICE IN CHILE
}

\begin{abstract}
The following work indicates the results of the application of 350 online surveys in the metropolitan region of Santiago de Chile between March 20 and April 20, 2019. Said survey was constructed and adapted based on the scales developed by (Zeithaml, Parasuraman and Malhotra; 2000), which were applied with the aim of collecting and analyzing the results obtained from the responses of consumers who made purchases through Amazon. The two scales proposed by the authors were applied; ES-Qual or main scale where the evaluated dimensions were: efficiency, system availability, compliance, privacy and the E-RecS-Qual scale that seeks to measure recovery or reestablishment when a customer has problems with the service, for the latter scale the dimensions were measured: perceived value, postsale and loyalty. This allowed us to visualize the perception of consumers as a result of the service provided by this company and thus to be able to visualize opportunities for improvement and growth for e-tailers in the Latin American context.
\end{abstract}

Keywords: e-tail, e-s-qual, e-rec-qual, on line service quality 


\section{INTRODUCCIÓN}

En el año 2000, los autores Zeithaml, Parasuraman y Malhotra identificaron docenas de funciones de los sitios web de empresas que comercializan productos en base a internet a nivel de atributo de percepción y las clasificaron en 11 dimensiones de e-SQ (e-Service Quality, se refiere a la calidad de servicio on line). A partir de este trabajo propusieron las escalas E-S-QUAL y E-Rec-QUAL, instrumentos diseñados para medir la percepción de calidad de los servicios on line. Dichas escalas han generado algunas críticas, siendo algunas de las principales, el número limitado de artículos académicos que tratan directamente sobre cómo los clientes evalúan e-SQ y la falta de validación y aplicación de la escala en distintas industrias y geografías.

Los autores consideran de gran importancia tanto para las organizaciones que ofrecen productos 0 servicio en base on line, como para el incremento del conocimiento académico local sobre esta temática, que este y otros instrumentos similares se validen, apliquen y se contribuya de esa forma a un mejor entendimiento sobre la percepción de calidad de las ventas on line considerando que en Chile, actualmente existen muy pocos estudios y mediciones al respecto. Las compañías necesitan de herramientas más poderosas dada la necesidad de mejorar los niveles de calidad percibida por el consumidor en el entorno actual de las ventas a distancia vía plataformas on line y especialmente necesario en el caso chileno con la entrada de Amazon (Modaes Latinoamerica; 2018) .

El resultado de las percepciones obtenidos refuerza también algunas teorías (Mick y Fournier; 1995) que indican que el proceso de compra en entornos virtuales es un proceso de largo plazo y que las empresas que decidan operar en dichos entornos, deberán plantearse 0 replantearse sus propuestas de valor para poder seguir operando exitosamente en el futuro.

El texto procederá como sigue. Primeramente, se expondrán los objetivos generales y específicos del estudio. El artículo prosigue con una breve revisión de la literatura relevante sobre el comercio detallista y la temática de la calidad de servicio. Luego de lo cual, se expondrá la metodología del estudio. En particular, se buscará definir con claridad el modelo y escalas de medición E-S-QUAL y E-Rec-QUAL y su aplicación en el fenómeno del comercio detallista. El texto prosigue con el análisis del caso de Amazon en el mercado chileno, para terminar, concluyendo acerca de la importancia de estudios sobre la calidad del servicio en entornos detallistas de carácter virtual.

\section{CONTRIBUCIONES}

A lo largo del presente trabajo, mostraremos la aplicación del constructo de e-SQ (e-Calidad de Servicio), compuesto por las escalas E-S-QUAL/E-Rec-QUAL y nos enfocaremos en el mercado chileno y en la calidad de servicio percibida de la 
empresa Amazon en el contexto de las compras on line. Además se observa: que es posible utilizar estos instrumentos para medir la e-SQ en Chile y en la industria a la que pertenece Amazon (retail), junto con las valoraciones que los consumidores en las distintas dimensiones que constituyen las escalas y las oportunidades de mejora que presentan las dimensiones menos valoradas.

\section{MARCO TEÓRICO}

Existen varios tipos de comercio on line que se diferencian por la naturaleza de los agentes implicados en la transacción. Ver Tabla 1.

Tabla 1.

\begin{tabular}{|c|c|}
\hline Comercio entre Empresas & Business to Business (B2B) \\
\hline Comercio entre Consumidores & Consumer to Consumer (C2C) \\
\hline Negocios entre Empresas y Consumidores & Business to Consumer (B2C) \\
\hline Comercio entre Amigos & Peer to Peer (P2P) \\
\hline Negocios entre Empresas y Empleados & Business to Employee (B2E) \\
\hline Comercio entre Gobiernos & Goverment to Goverment (G2G) \\
\hline
\end{tabular}

Siendo el concepto genérico de e-tail una contracción de e-retailing y representa una forma de comercio electrónico que permite a los consumidores comprar directamente bienes o servicios de un vendedor a través de Internet. Este concepto fue definido por (Jones et al; 2001) como "el aprovisionamiento, la compra / venta y el pago de bienes y servicios en los cuales el proceso interactivo está mediado por información o tecnología digital en ambos extremos del intercambio localmente separados". Mientras que varios años después (Sarkar \& Das;2016) definen e-retail o e-tail como "la venta de bienes y servicios a través de Internet u otros canales electrónicos, para uso personal o doméstico por parte de los consumidores". El foco de nuestra investigación se refiere a una empresa que vende productos o proporciona servicios a los consumidores finales, (B2C) que (Laudon y Traver;2014), al respecto realizan una definición más precisa, donde incluyen compras de bienes al por menor, servicios de viaje y contenido en línea.

Desde el punto de vista teórico/práctico esta manera de comercializar de acuerdo a (Cross \& Smith;1995) beneficia a los consumidores, los cuales pueden hacer comparaciones en sus compras al tener una buena información de los productos y servicios, la disponibilidad de ellos en las tiendas, pudiendo acceder a ellos (Bakos;1997) con precios más convenientes pudiendo hacer un seguimiento en línea del proceso de compra completo, incluyendo el servicio postventa. Por otro lado, los vendedores pueden beneficiarse de menores gastos relacionados con infraestructura (Schlauch \& Laposa; 2001) y mejor gestión a través de los estudios de mercado (Murphy et al.; 2001). A pesar de ello, los consumidores a veces les resulta difícil navegar por ciertos sitios y muchas veces se desilusionan por encontrarlos engorrosos y lentos. Al respecto hay una serie de investigaciones que de alguna manera develan estos inconvenientes. 


\section{CALIDAD DE SERVICIO ON LINE (e-SQ)}

Cada día, un mayor número de compañías intensifican su presencia on line para realizar la venta de sus productos 0 servicios. Para que este canal de venta sea viable, debe ser percibido por parte de los consumidores como efectivo y eficiente (Zeithaml, Parasuraman y Malhotra; 2000). Inicialmente se pensaba que la presencia en la Web y los precios bajos constituían las principales razones de éxito, no obstante, en cuanto se manifestaron diversos inconvenientes tales como que: los consumidores no podían completar sus transacciones, los productos no llegaban a tiempo o simplemente no llegaban, los canales de comunicación consumidor/empresa no funcionaban, la calidad de servicio on line se transformó en fundamental. Sendas investigaciones pusieron en evidencia los problemas mencionados anteriormente, donde podemos destacar a los desarrollados por (Ahmad 2002; Lennon y Harris 2002; LoCascio 2000; Pastore 2001;Cox 2002; Gaudin 2003; InternetNewsBureau 2003).

Es por ello que la percepción de calidad de servicio on line (e-SQ), se hace una de las variables fundamentales a la hora de gestionar compañías en base Web, tomando en consideración que de acuerdo a distintos autores, como (Parasuraman, et al., 2005) y (Mick and Fournier, 1995) sugieren que: "(a) la satisfacción del cliente con tales productos implica un proceso altamente complejo, cargado de significado, a largo plazo; (b) el proceso puede variar entre diferentes segmentos de clientes; $y$ (c) la satisfacción en tales contextos no siempre es una función de Ios estándares de comparación de preconsumo". La calidad del servicio online (e-SQ) se transforma en una de las variables centrales del altamente complejo proceso de satisfacción del cliente.

Consecuentemente, consideramos de suma importancia aplicar instrumentos fiables para medir la calidad de servicio on line (e-SQ), mismos que si bien es cierto, no están desarrollados para el contexto latinoamericano, será posible y necesario validarlos y adaptarlos para de esa manera, midiendo la calidad de servicio (on line), sirvan a las compañías que promueven las ventas en línea.

\section{MODELOS de e-SQ (E-S-QUAL y E-Rec-QUAL)}

La primera escala desarrollada que capturó efectivamente la naturaleza de la calidad del servicio electrónico desde la perspectiva de las compras en línea a través de un sitio Web minorista fue la escala E-S-QUAL, que consideraba una escala principal (E-S-QUAL) y una segunda escala desarrollada por los autores, la cual pretende medir el restablecimiento o la recuperación (E-RecS-QUAL), cuando algún cliente presenta algún inconveniente con el servicio.

Por medio del modelo E-S-QUAL tradicional, nos referimos a una comparación de lo que los clientes consideran que una empresa debería ofrecer (sus expectativas) con el rendimiento real del servicio de la empresa calidad de todas las interacciones 
y experiencias con clientes que no están basadas en Internet y la escala E-RecSQUAL se utiliza para evaluar la post venta o recuperación (Sasser, Olsen y Wyckoff, 1978; Grönroos;1982; Lehtinen y Lehtinen ,1982; Lewis y Booms 1983; Parasuraman, Zeithaml y Berry, 1985) Este artículo utiliza la escala de elementos múltiples E-S-QUAL, para medir la calidad del servicio entregado por los sitios web para aquellos clientes que compran en línea en Chile, A pesar de que han existido diversos acercamientos para medir la calidad (Watson Goodhue,2000) creó WebQual, (Barnes y Vidgen, 2002) desarrollaron otra proposición de escala, para medir lo mismo, un año antes (Yoo y Donthu;2001) crearon una escala denominada SITEQUAL (Wolfinbarger y Gilly;2003) por su parte, utilizaron grupos focales en línea y fuera de línea, en otras palabras, diversos intentos por medir la calidad. Con todo, la escala referida E-S-QUAL una de sus principales críticas, es justamente, el número limitado de artículos académicos que tratan directamente sobre cómo los clientes evalúan e-SQ, no obstante, quienes han aplicado la escala sugieren que puede dar buenos resultados (Santouridis et al. 2012; Yaya et al., 2011; Rafiq et al., 2011; Boshoff, 2007; Connolly, 2007). La información obtenida de los estudios que tratan sobre las interacciones entre personas y tecnología implica que la evaluación de nuevas tecnologías por parte de los clientes es un proceso distinto. (Mick y Fournier 1995; 1998). En especial, lo relativo a los sentimientos positivos y negativos que se desencadenan simultáneamente con el uso de la tecnología (Parasuraman; 2000).

La escala también suma otras criticas de autores como (Bauer et al., 2006) que señala la ausencia de componentes hedónicos en el modelo y sugiere su inclusión. No obstante la escala fue validada por Yang y Tsai (2007) y concluye que es valido para determinar la lealtad on-line. A su vez (Park y Kim, 2003) en sus investigaciones señalan que la confianza y el compromiso cliente/empresa, consideradas en la escala determinan la lealtad.

\section{METODOLOGÍA}

La investigación se basó en el estudio de (Zeithaml, Parasuraman y Malhotra; 2000). Para aplicar el constructo obtenido por (Zeithaml, Parasuraman y Malhotra ;2000), se procedió a adaptarlo al lenguaje castellano como se puede ver en el anexo $n^{\circ 1}$ y posteriormente se aplicó vía Internet mediante un servicio de mail marketing con soporte de un formulario Google la encuesta de 39 ítems (representando las dimensiones de E-S-QUAL y E-REc-QUAL), con opción de respuesta en escala Likert, en la región metropolitana de Chile entre el 20 de marzo y el 20 de abril de 2019. Una vez enviado el formulario con la encuesta se obtuvieron (por conveniencia, esto significa que se recopilaron respuestas de personas que estuvieron dispuestas a responderla), 350 respuestas correctas, luego de ser rechazadas todas aquellas respuestas que se presentaban incompletan o presentaban algún tipo de defecto.

En el proceso de adaptación del instrumento, se obtuvieron las siguientes dimensiones y preguntas para aplicar a los encuestados. Ver Tabla 2. 
Tabla 2. Dimensiones y Preguntas del Instrumento

\begin{tabular}{|c|c|}
\hline \multirow{8}{*}{ Eficiencia } & EFF1 Este sitio hace que sea fácil encontrar lo que necesito. \\
\hline & EFF2 Hace que sea fácil llegar a cualquier parte del sitio. \\
\hline & EFF3 Me permite completar una transacción rápidamente. \\
\hline & EFF4 La información en este sitio está bien organizada. \\
\hline & EFF5 Carga sus páginas rápido. \\
\hline & EFF6 Este sitio es fácil de usar. \\
\hline & EFF7 Este sitio me permite acceder a él rápidamente. \\
\hline & EFF8 Este sitio está bien organizado. \\
\hline \multirow{4}{*}{$\begin{array}{c}\text { Disponibilidad } \\
\text { del Sistema }\end{array}$} & SYS1 Este sitio 5 está disponible para Operar. \\
\hline & SYS2 Este sitio se inicia y se ejecuta de inmediato. \\
\hline & SYS3 Este sitio se bloquea. \\
\hline & $\begin{array}{l}\text { SYS4 Las páginas de este sitio se quedan "pegadas o congeladas" } \\
\text { después de ingresar el pedido. }\end{array}$ \\
\hline \multirow{3}{*}{ Privacidad } & $\begin{array}{l}\text { PRI1 Protege la información sobre mi comportamiento de compra en la } \\
\text { web. }\end{array}$ \\
\hline & PRI2 Este sitio comparte mi información personal con otros sitios. \\
\hline & $\begin{array}{l}\text { PRI3 Este sitio protege información sobre mi tarjeta de crédito o medio } \\
\text { de pago. }\end{array}$ \\
\hline \multirow{7}{*}{ Cumplimiento } & FUL1 Entrega las órdenes cuando se prometió. \\
\hline & $\begin{array}{l}\text { FUL2 Este sitio hace que los artículos estén disponibles para su entrega } \\
\text { en un tiempo adecuado }\end{array}$ \\
\hline & FUL3 Entrega rápidamente lo que ordeno. \\
\hline & FUL4 Envía los artículos ordenados. \\
\hline & FUL5 tiene en stock los artículos que la compañía afirma tener. \\
\hline & FUL6 Es veraz sobre sus ofertas. \\
\hline & FUL7 Hace promesas precisas sobre la entrega de productos. \\
\hline \multirow{4}{*}{$\begin{array}{l}\text { Valor } \\
\text { Percibido }\end{array}$} & VPE1 Los precios de los productos y servicios disponibles en el sitio. \\
\hline & VPE2 La conveniencia general de usar este sitio. \\
\hline & VPE3 La medida en que el sitio te da una sensación de tener el control. \\
\hline & VPE4 El valor total que obtiene de este sitio por su dinero y esfuerzo. \\
\hline \multirow{11}{*}{ Post Venta } & RES1 Me proporciona opciones convenientes para devolver artículos. \\
\hline & RES2 Este sitio maneja bien las devoluciones de productos. \\
\hline & RES3 Este sitio ofrece una garantía significativa. \\
\hline & RES4 Me dice qué hacer si mi transacción no es procesada. \\
\hline & RES5 Se ocupa de los problemas a la brevedad. \\
\hline & COM1 Este sitio me compensa por los problemas que crea. \\
\hline & COM2 Me compensa cuando lo que ordené no llega a tiempo. \\
\hline & COM3 Recoge los artículos que quiero devolver de mi casa o negocio. \\
\hline & $\begin{array}{l}\text { CON1 Este sitio proporciona un número de teléfono para acceder a la } \\
\text { empresa. }\end{array}$ \\
\hline & $\begin{array}{l}\text { CON2 Este sitio tiene representantes de servicio al cliente disponibles } \\
\text { en línea. }\end{array}$ \\
\hline & $\begin{array}{l}\text { CON3 Ofrece la posibilidad de hablar con una persona viva si existe un } \\
\text { problema. }\end{array}$ \\
\hline
\end{tabular}




\begin{tabular}{|l|l|}
\hline \multirow{y}{*}{ Lealtad } & $\begin{array}{l}\text { LEA1 ¿Qué tan probable es que usted diga cosas positivas sobre este } \\
\text { sitio a otras personas? }\end{array}$ \\
\cline { 2 - 2 } & $\begin{array}{l}\text { LEA2 ¿Qué tan probable es que usted recomiende este sitio a alguien } \\
\text { que busca tu consejo? }\end{array}$ \\
\cline { 2 - 2 } & $\begin{array}{l}\text { LEA3 ¿Qué tan probable es que usted anime a amigos y otras personas } \\
\text { a hacer compras en este sitio? }\end{array}$ \\
\cline { 2 - 2 } & $\begin{array}{l}\text { LEA4 ¿Qué tan probable es que usted considere a este sitio como su } \\
\text { primera opción para futuras transacciones? }\end{array}$ \\
\hline & $\begin{array}{l}\text { LEA5 ¿Qué tan probable es que usted haga más compras en este sitio } \\
\text { en los próximos meses? }\end{array}$ \\
\hline
\end{tabular}

\section{RESULTADOS}

A continuación se analizan los resultados de las 350 respuestas correctas a la encuesta, de las cuales se puede destacar los siguientes datos:

- $\quad$ Del total encuestados, un $44.87 \%$ (157) corresponden al sexo femenino y un $55,14 \%$ (193) al sexo masculino.

- El medio de pago de mayor uso corresponde a la tarjeta de crédito bancaria con un $44,68 \%$ y le siguen el pago con tarjeta de débito con un $38,30 \%$ y otros medios de pago con un $17,02 \%$.

- $\quad$ El $75 \%$ de los encuestados tiene educación superior completa 0 incompleta.

- $\quad$ El grupo familiar promedio de los encuestados es de 4 personas $(4,41)$.

Posteriormente se revisan los resultados obtenidos de la percepción sobre Amazon en las distintas dimensiones del constructo E-S-QUAL y E-Rec-QUAL:

Tabla 3. Valores Promedio de las Dimensiones y Máxima Calificación Posible.

\begin{tabular}{|c|c|c|c|c|c|c|c|}
\hline $\begin{array}{c}\text { Portal o } \\
\text { Empresa }\end{array}$ & Eficiencia & Disponibilidad & Privacidad & Cumplimiento & $\begin{array}{c}\text { Valor } \\
\text { Percibido }\end{array}$ & $\begin{array}{c}\text { Post } \\
\text { Venta }\end{array}$ & Lealtad \\
\hline Amazon & 4,39 & 3,46 & 4,08 & 4,23 & 5,65 & 3,73 & $\mathbf{4 , 0 8}$ \\
\hline $\begin{array}{c}\text { Calificación } \\
\text { Máxima } \\
\text { Posible }\end{array}$ & 5,00 & 5,00 & 5,00 & 5,00 & 7,00 & 5,00 & 5,00 \\
\hline
\end{tabular}

Fuente: elaboración propia 


\section{RESULTADOS DE LA DIMENSIÓN EFICIENCIA}

En la dimensión de eficiencia, Amazon obtiene una puntuación promedio de 4.39, encontrando la mayor diferencia respecto a la máxima puntuación posible en el atributo EFF1 y EFF2 que corresponden a la facilidad de búsqueda y la facilidad para llegar a las distintas secciones de la página web de la empresa.

Tabla 4. Estadísticos Atributos de Eficiencia (EFF).

\begin{tabular}{|c|c|c|c|c|c|c|c|}
\hline \multicolumn{7}{|c|}{ Estadísticos Atributos de Eficiencia (EFF) } \\
\hline & $N$ & Rango & Mínimo & Máximo & Media & $\begin{array}{c}\text { Desv. } \\
\text { Desviación }\end{array}$ & Varianza \\
\hline $\begin{array}{c}\text { EFF1 Este sitio } \\
\text { hace que sea fácil } \\
\text { encontrar lo que } \\
\text { necesito. }\end{array}$ & 350 & 2,00 & 3,00 & 5,00 & 4,2084 &, 80010 &, 640 \\
\hline $\begin{array}{c}\text { EFF2 Hace que } \\
\text { sea fácil llegar a } \\
\text { cualquier parte del } \\
\text { sitio. }\end{array}$ & 350 & 2,00 & 3,00 & 5,00 & 4,1251 &, 71165 &, 506 \\
\hline $\begin{array}{c}\text { EFF3 Me permite } \\
\text { completar una } \\
\text { transacción } \\
\text { rápidamente. }\end{array}$ & 350 & 2,00 & 3,00 & 5,00 & 4,2497 &, 81357 &, 662 \\
\hline $\begin{array}{c}\text { EFF4 La } \\
\text { información en } \\
\text { este sitio está bien } \\
\text { organizada. }\end{array}$ & 350 & 2,00 & 3,00 & 5,00 & 4,5415 &, 69261 &, 480 \\
\hline $\begin{array}{c}\text { EFF5 Carga sus } \\
\text { páginas rápido. }\end{array}$ & 350 & 2,00 & 3,00 & 5,00 & 4,3335 &, 61189 &, 374 \\
\hline $\begin{array}{c}\text { EFF6 Este sitio es } \\
\text { fácil de usar. }\end{array}$ & 350 & 2,00 & 3,00 & 5,00 & 4,6248 &, 62137 &, 386 \\
\hline $\begin{array}{c}\text { EFF7 Este sitio me } \\
\text { permite acceder a } \\
\text { él rápidamente. }\end{array}$ & 350 & 2,00 & 3,00 & 5,00 & 4,6253 &, 62137 &, 386 \\
\hline $\begin{array}{c}\text { EFF8 Este } \\
\text { sitio está bien } \\
\text { organizado. }\end{array}$ & 350 & 3,00 & 2,00 & 5,00 & 4,3747 &, 84085 &, 707 \\
\hline N válido (por lista) & 350 & & & & & & \\
\hline
\end{tabular}

Fuente: Elaboración Propia 
Figura 1. Promedio de los Atributos de Eficiencia.

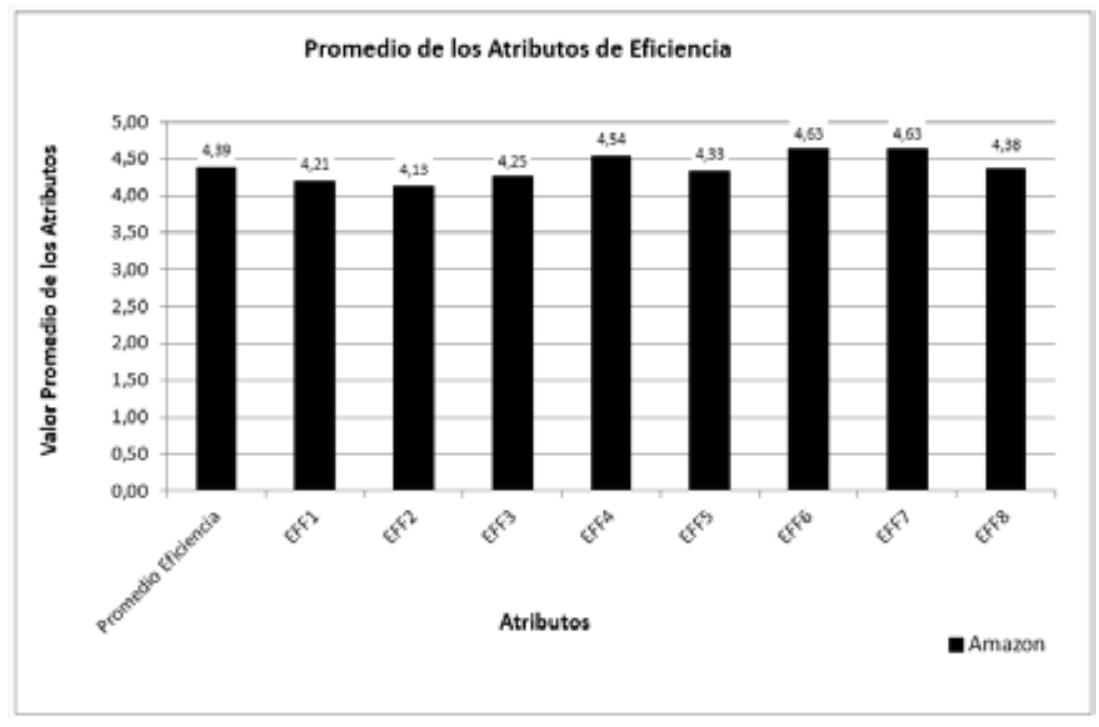

Fuente: Elaboración Propia

\section{RESULTADOS DE LA DIMENSIÓN DISPONIBILIDAD DEL SISTEMA}

En la dimensión de disponibilidad del sistema, Amazon obtiene un promedio de 3.46, encontrando la mayor distancia respecto de la máxima calificación posible en los atributos SYS3 y SYS4, que se refieren al bloqueo de la página y a que estás se queden eventualmente "pegadas" impidiendo realizar una transacción (con una calificación de 2.33 en ambos casos).

Tabla 5. Estadísticos Atributos Disponibilidad del Sistema (SYS).

\begin{tabular}{|c|c|c|c|c|c|c|c|}
\hline \multicolumn{7}{|c|}{ Estadísticos Atributos Disponibilidad del Sistema (SYS) } \\
\hline & $\mathrm{N}$ & Rango & Mínimo & Máximo & Media & $\begin{array}{c}\text { Desv. } \\
\text { Desviación }\end{array}$ & Varianza \\
\hline $\begin{array}{c}\text { SYS1 Este sitio } \\
5 \text { está disponible } \\
\text { para Operar. }\end{array}$ & 350 & 2,00 & 3,00 & 5,00 & 4,5415 &, 63204 &, 399 \\
\hline $\begin{array}{c}\text { SYS2 Este sitio se } \\
\text { inicia y se ejecuta } \\
\text { de inmediato. }\end{array}$ & 350 & 2,00 & 3,00 & 5,00 & 4,6248 &, 62137 &, 386 \\
\hline $\begin{array}{c}\text { SYS3 Este sitio se } \\
\text { bloquea. }\end{array}$ & 350 & 4,00 & 1,00 & 5,00 & 2,3326 & 1,25613 & 1,578 \\
\hline
\end{tabular}




\begin{tabular}{|c|c|c|c|c|c|c|c|}
\hline $\begin{array}{c}\text { SYS4 Las páginas } \\
\text { de este sitio se } \\
\text { quedan "pegadas } \\
\text { o congeladas" } \\
\text { después de } \\
\text { ingresar a mi } \\
\text { información del } \\
\text { pedido. }\end{array}$ & 350 & 4,00 & 1,00 & 5,00 & 2,3326 & 1,37796 & 1,899 \\
\hline N válido (por lista) & 350 & & & & & & \\
\hline
\end{tabular}

Fuente: Elaboración Propia

Figura 2. Promedio de los Atributos de Disponibilidad del Sistema.

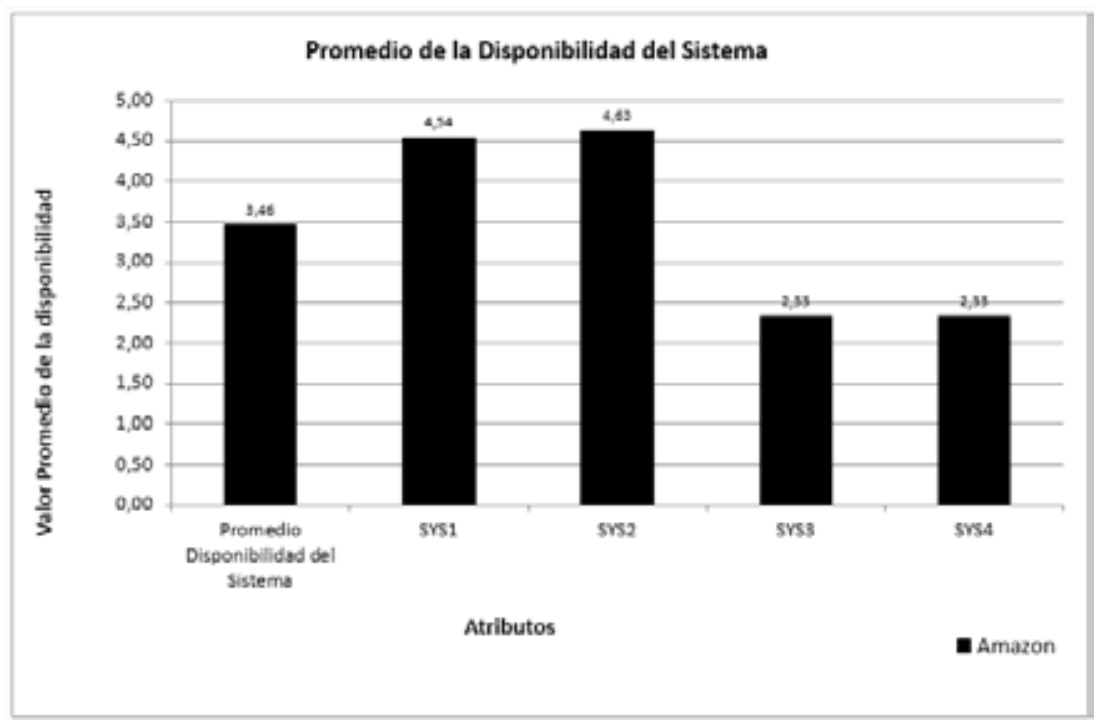

Fuente: Elaboración Propia

\section{RESULTADOS DE LA DIMENSIÓN PRIVACIDAD}

Respecto a la privacidad, Amazon promedia un 4.08 y se observa una mayor distancia en el atributo PRI2 respecto a la máxima calificación posible (PRI2 se refiere a la percepción de que la empresa comparte los datos personales con otras páginas 0 aplicaciones). 
Tabla 6. Estadísticos Atributos de Privacidad

\begin{tabular}{|c|c|c|c|c|c|c|c|}
\hline \multicolumn{7}{|c|}{ Estadísticos Atributos de Privacidad } \\
\hline & N & Rango & Mínimo & Máximo & Media & $\begin{array}{c}\text { Desv. } \\
\text { Desviación }\end{array}$ & Varianza \\
\hline $\begin{array}{c}\text { PRI1 Protege la } \\
\text { información sobre } \\
\text { mi comportamiento } \\
\text { de compra en la } \\
\text { web. }\end{array}$ & 350 & 3,00 & 2,00 & 5,00 & 4,1667 & 1,08476 & 1,177 \\
\hline $\begin{array}{c}\text { PRI2 Este sitio } \\
\text { comparte mi } \\
\text { información personal } \\
\text { con otros sitios. }\end{array}$ & 350 & 4,00 & 1,00 & 5,00 & 3,3752 & 1,54656 & 2,392 \\
\hline $\begin{array}{c}\text { PRI3 Este sitio } \\
\text { protege información } \\
\text { sobre mi tarjeta de } \\
\text { crédito o medio de } \\
\text { pago. }\end{array}$ & 350 & 3,00 & 2,00 & 5,00 & 4,7082 &, 72098 &, 520 \\
\hline N válido (por lista) & 350 & & & & & & \\
\hline
\end{tabular}

Fuente: Elaboración Propia

Figura 3. Promedio de los Atributos de Privacidad.

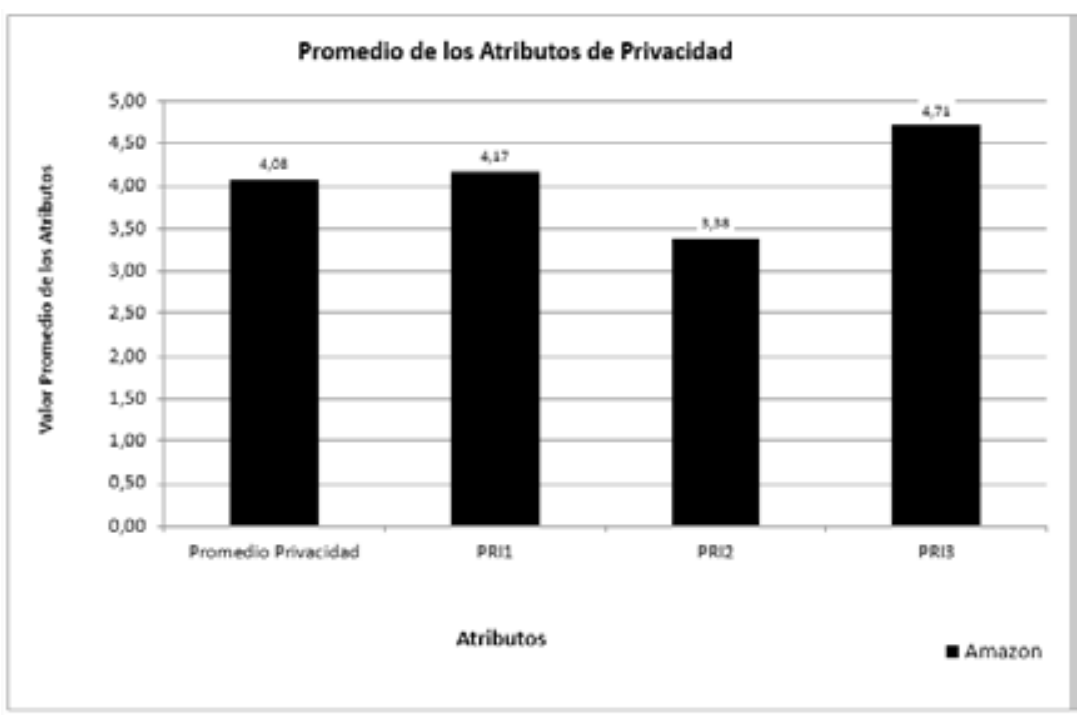

Fuente: Elaboración Propia 


\section{RESULTADOS DE LA DIMENSIÓN CUMPLIMIENTO}

La percepción de los consumidores respecto a la dimensión de cumplimiento para Amazon, promedia 4.25, encontrando en los atributos FUL1 y FUL3 una mayor distancia respecto de la máxima calificación posible (FUL1 se refiere a que la empresa entrega lo ordenado cuando se compromete y FUL3 se refiere a la rapidez de la entrega).

Tabla 7: Estadísticos Atributos de Cumplimiento (FUL).

\begin{tabular}{|c|c|c|c|c|c|c|c|}
\hline \multicolumn{7}{|c|}{ Estadísticos Atributos de Cumplimiento (FUL) } \\
\hline & N & Rango & Mínimo & Máximo & Media & $\begin{array}{c}\text { Desv. } \\
\text { Desviación }\end{array}$ & Varianza \\
\hline $\begin{array}{c}\text { FUL1 Entrega las } \\
\text { órdenes cuando se } \\
\text { prometió. }\end{array}$ & 350 & 3,00 & 2,00 & 5,00 & 3,9587 &, 91692 &, 841 \\
\hline $\begin{array}{c}\text { FUL2 Este sitio hace } \\
\text { que los artículos } \\
\text { estén disponibles } \\
\text { para su entrega } \\
\text { dentro de un marco } \\
\text { de tiempo adecuado. }\end{array}$ & 350 & 3,00 & 2,00 & 5,00 & 4,2926 &, 72102 &, 520 \\
\hline $\begin{array}{c}\text { FUL3 Entrega } \\
\text { rápidamente lo que } \\
\text { ordeno. }\end{array}$ & 350 & 2,00 & 3,00 & 5,00 & 4,0004 &, 69382 &, 481 \\
\hline $\begin{array}{c}\text { FUL4 Envía los } \\
\text { artículos ordenados. }\end{array}$ & 350 & 3,00 & 2,00 & 5,00 & 4,4173 &, 89198 &, 796 \\
\hline $\begin{array}{c}\text { FUL5 tiene en stock } \\
\text { los artículos que la } \\
\text { compañía afirma } \\
\text { tener. }\end{array}$ & 350 & 2,00 & 3,00 & 5,00 & 4,5006 &, 69383 &, 481 \\
\hline $\begin{array}{c}\text { FUL6 Es veraz sobre } \\
\text { sus ofertas. }\end{array}$ & 350 & 2,00 & 3,00 & 5,00 & 4,3339 &, 73135 &, 535 \\
\hline $\begin{array}{c}\text { FUL7 Hace } \\
\text { promesas precisas } \\
\text { sobre la entrega de } \\
\text { productos. }\end{array}$ & 350 & 3,00 & 2,00 & 5,00 & 4,1246 &, 95268 &, 908 \\
\hline N válido (por lista) & 350 & & & & & & \\
\hline
\end{tabular}

Fuente: Elaboración Propia 
Figura 4. Promedio de los Atributos de Cumplimiento.

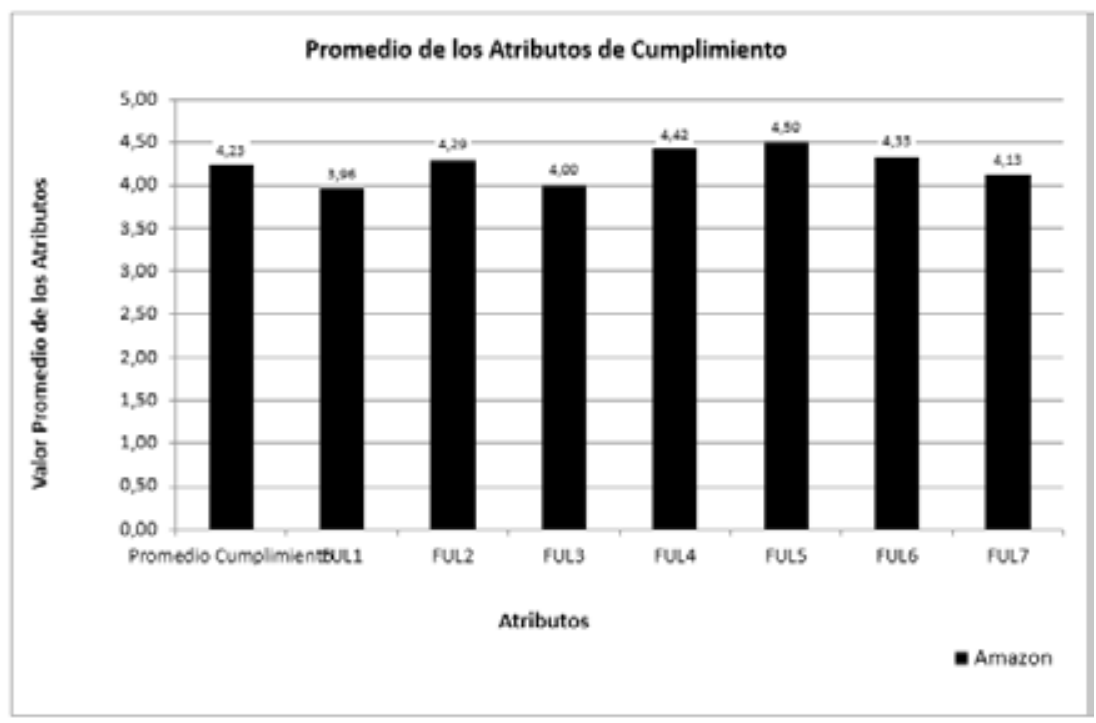

Fuente: Elaboración Propia

\section{RESULTADOS DE LA DIMENSIÓN VALOR PERCIBIDO}

En la dimensión de Valor Percibido, Amazon obtiene un promedio de 5.65, presentándose valores similares al promedio en todos los atributos de la dimensión.

Tabla 8: Estadísticos Atributos de Valor Percibido (VPE).

\begin{tabular}{|c|c|c|c|c|c|c|c|}
\hline \multicolumn{7}{|c|}{ Estadísticos Atributos de Valor Percibido (VPE) } \\
\hline & N & Rango & Mínimo & Máximo & Media & $\begin{array}{c}\text { Desv. } \\
\text { Desviación }\end{array}$ & Varianza \\
\hline $\begin{array}{c}\text { VPE1 Los precios } \\
\text { de los productos y } \\
\text { servicios disponibles } \\
\text { en el sitio. }\end{array}$ & 350 & 6,00 & 1,00 & 7,00 & 5,3752 & 1,46668 & 2,151 \\
\hline $\begin{array}{c}\text { VPE2 La } \\
\text { conveniencia general } \\
\text { de usar este sitio. }\end{array}$ & 350 & 4,00 & 3,00 & 7,00 & 5,8333 & 1,08476 & 1,177 \\
\hline $\begin{array}{c}\text { VPE3 La medida en } \\
\text { que el sitio te da una } \\
\text { sensación de tener } \\
\text { el control. }\end{array}$ & 350 & 4,00 & 3,00 & 7,00 & 5,8758 & 1,36762 & 1,870 \\
\hline $\begin{array}{c}\text { VPE4 El valor total } \\
\text { que obtiene de este } \\
\text { sitio por su dinero y } \\
\text { esfuerzo. }\end{array}$ & 350 & 6,00 & 1,00 & 7,00 & 5,5006 & 1,38763 & 1,926 \\
\hline N válido (por lista) & 350 & & & & & & \\
\hline
\end{tabular}

Fuente: Elaboración Propia 
Figura 5. Promedio de los Atributos de Valor Percibido.

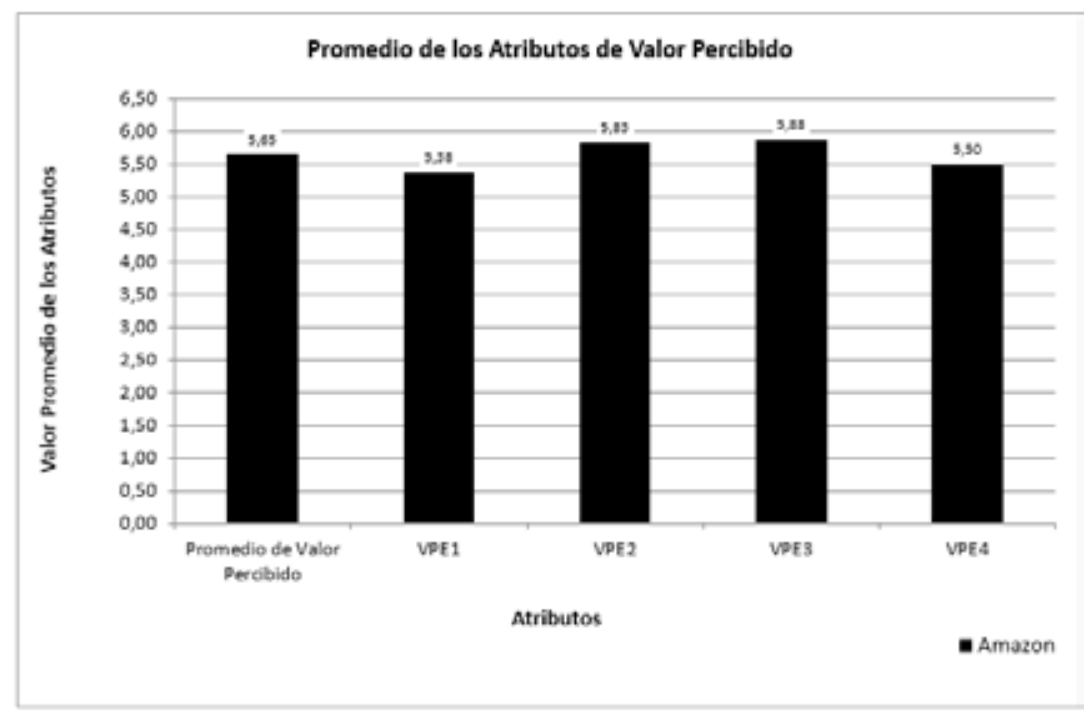

Fuente: Elaboración Propia

\section{RESULTADOS DE LA DIMENSIÓN POST VENTA}

Los datos arrojados para la dimensión de post venta, Amazon presenta un promedio del atributo de 3.73, encontrando que en los atributos RES2 (manejo de las devoluciones de productos), COM2 (compensación al consumidor cuando la orden no llega a tiempo) y COM3 (Recogida de las devoluciones en el domicilio del consumidor), se presentan las mayores distancias en relación a la máxima calificación posible.

Tabla 9. Estadísticos Atributos de Post Venta (RES, COM, CON).

\begin{tabular}{|c|c|c|c|c|c|c|c|}
\hline \multicolumn{7}{|c|}{ Estadísticos Atributos de Post Venta (RES, COM, CON) } \\
\hline & N & Rango & Mínimo & Máximo & Media & $\begin{array}{c}\text { Desv. } \\
\text { Desviación }\end{array}$ & Varianza \\
\hline $\begin{array}{c}\text { COM1 Este sitio me } \\
\text { compensa por los } \\
\text { problemas que crea. }\end{array}$ & 350 & 4,00 & 1,00 & 5,00 & 3,5836 & 1,23194 & 1,518 \\
\hline $\begin{array}{c}\text { COM2 Me } \\
\text { compensa cuando lo } \\
\text { que ordené no llega } \\
\text { a tiempo. }\end{array}$ & 350 & 4,00 & 1,00 & 5,00 & 3,4585 & 1,32792 & 1,763 \\
\hline $\begin{array}{c}\text { COM3 Recoge los } \\
\text { artículos que quiero } \\
\text { devolver de mi casa } \\
\text { 0 negocio. }\end{array}$ & 350 & 4,00 & 1,00 & 5,00 & 3,3335 & 1,22377 & 1,498 \\
\hline
\end{tabular}




\begin{tabular}{|c|c|c|c|c|c|c|c|}
\hline $\begin{array}{l}\text { CON1 Este sitio } \\
\text { proporciona un } \\
\text { número de teléfono } \\
\text { para acceder a la } \\
\text { empresa. }\end{array}$ & 350 & 4,00 & 1,00 & 5,00 & 3,7086 & 1,21761 & 1,483 \\
\hline $\begin{array}{l}\text { CON2 Este sitio tiene } \\
\text { representantes de } \\
\text { servicio al cliente } \\
\text { disponibles en linea. }\end{array}$ & 350 & 4,00 & 1,00 & 5,00 & 3,8337 & 1,15636 & 1,337 \\
\hline $\begin{array}{l}\text { CON3 Ofrece la } \\
\text { posibilidad de hablar } \\
\text { con una persona } \\
\text { viva (no un sistema } \\
\text { automatizado), si } \\
\text { existe un problema. }\end{array}$ & 350 & 4,00 & 1,00 & 5,00 & 3,5836 & 1,29543 & 1,678 \\
\hline $\begin{array}{c}\text { RES1 Me } \\
\text { proporciona } \\
\text { opciones } \\
\text { convenientes para } \\
\text { devolver artículos. }\end{array}$ & 350 & 3,00 & 2,00 & 5,00 & 3,8749 & ,90960 & ,827 \\
\hline $\begin{array}{l}\text { RES2 Este sitio } \\
\text { maneja bien las } \\
\text { devoluciones de } \\
\text { productos. }\end{array}$ & 350 & 3,00 & 2,00 & 5,00 & 3,5836 & 1,05666 & 1,117 \\
\hline $\begin{array}{l}\text { RES3 Este sitio } \\
\text { ofrece una garantía } \\
\text { significativa. }\end{array}$ & 350 & 3,00 & 2,00 & 5,00 & 3,8337 & 1,04713 & 1,096 \\
\hline $\begin{array}{l}\text { RES4 Me dice } \\
\text { qué hacer si mi } \\
\text { transacción no es } \\
\text { procesada. }\end{array}$ & 350 & 4,00 & 1,00 & 5,00 & 4,2088 & 1,13226 & 1,282 \\
\hline $\begin{array}{l}\text { RES5 Se ocupa de } \\
\text { los problemas a la } \\
\text { brevedad. }\end{array}$ & 350 & 3,00 & 2,00 & 5,00 & 4,0004 & ,89571 & ,802 \\
\hline $\mathrm{N}$ válido (por lista) & 350 & & & & & & \\
\hline
\end{tabular}

Fuente: Elaboración Propia 
Figura 6. Promedio de los Atributos de Post Venta.

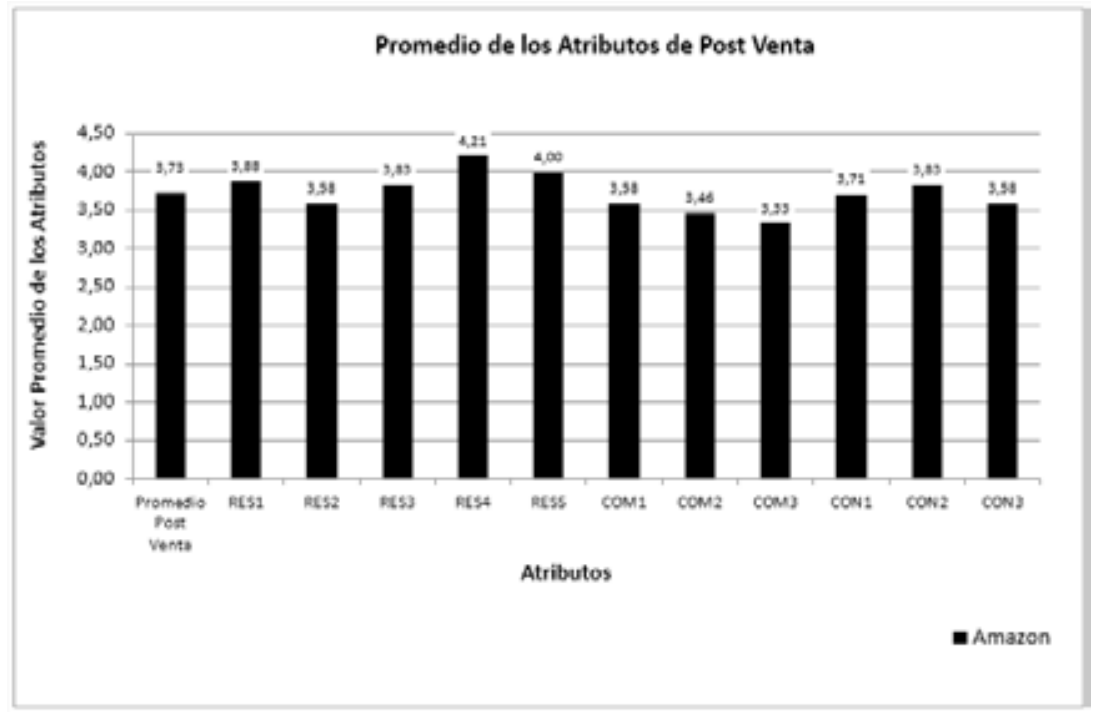

Fuente: Elaboración Propia

\section{RESULTADOS DE LA DIMENSIÓN LEALTAD}

El resultado promedio en la dimensión lealtad fue de 4.08, presentando en general buenos valores en todos los atributos, pero observamos en los atributos LEA2 (recomendación a otros el sitio web) y LEA5 (la probabilidad de realizar más compras en el futuro en el mismo sitio) un valor más por debajo que el resto de los atributos; 3.88 y 3.67 respectivamente.

Tabla 10. Estadísticos Atributos de Lealtad (LEA).

\begin{tabular}{|c|c|c|c|c|c|c|c|}
\hline \multicolumn{7}{|c|}{ Estadísticos Atributos de Lealtad (LEA) } \\
\hline & N & Rango & Mínimo & Máximo & Media & $\begin{array}{c}\text { Desv. } \\
\text { Desviación }\end{array}$ & Varianza \\
\hline $\begin{array}{c}\text { LEA1 iQué tan } \\
\text { probable es que usted } \\
\text { diga cosas positivas } \\
\text { sobre este sitio a } \\
\text { otras personas? }\end{array}$ & 350 & 3,00 & 2,00 & 5,00 & 4,2501 &, 76267 &, 582 \\
\hline $\begin{array}{c}\text { LEA2 iQué tan } \\
\text { probable es que usted } \\
\text { recomiende este sitio } \\
\text { a alguien que busca } \\
\text { tu consejo? }\end{array}$ & 350 & 2,00 & 3,00 & 5,00 & 4,3335 &, 78428 &, 615 \\
\hline
\end{tabular}




\begin{tabular}{|c|l|l|l|l|l|l|l|}
\hline $\begin{array}{c}\text { LEA3 iQué tan } \\
\text { probable es que usted } \\
\text { anime a amigos y } \\
\text { otras personas a } \\
\text { hacer compras en } \\
\text { este sitio? }\end{array}$ & 350 & 3,00 & 2,00 & 5,00 & 3,8749 &, 81664 &, 667 \\
\hline $\begin{array}{c}\text { LEA4 ¿Qué tan } \\
\text { probable es que usted } \\
\text { considere a este sitio } \\
\text { como su primera } \\
\text { opción para futuras } \\
\text { transacciones? }\end{array}$ & 350 & 2,00 & 3,00 & 5,00 & 4,2501 &, 64900 &, 421 \\
\hline $\begin{array}{c}\text { LEA5 iQué tan } \\
\text { probable es que usted } \\
\text { haga más compras } \\
\text { en este sitio en los } \\
\text { próximos meses? }\end{array}$ & 350 & 4,00 & 1,00 & 5,00 & 3,6665 &, 92508 &, 856 \\
\hline Nálido (por lista) & 350 & & & & & & \\
\hline
\end{tabular}

Fuente: Elaboración Propia

Figura 7. Promedio de los Atributos de Lealtad.

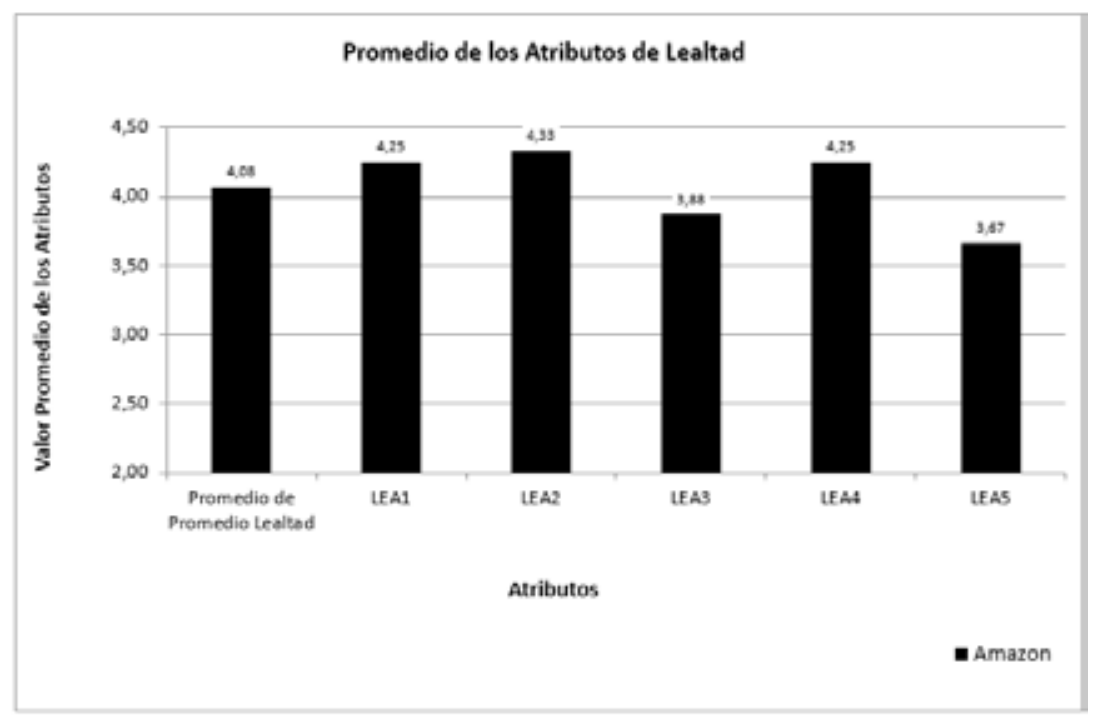

Fuente: Elaboración Propia

\section{RESULTADOS GENERALES}

Los resultados generales comparados con las máximas calificaciones posibles (por parte de los consumidores), indican que existen muchas oportunidades de mejora para obtener una mejor experiencia de usuario y con ello fidelidad en el 
largo plazo. Se puede destacar 2 dimensiones en las cuales Amazon presenta las mayores distancias; la dimensión de disponibilidad del sistema y la post venta.

En la dimensión de disponibilidad del sistema, Amazon obtiene un promedio de 3.46 , encontrando la mayor distancia respecto de la máxima calificación posible en los atributos SYS3 y SYS4, que se refieren al bloqueo de la página y a que estás se queden eventualmente "pegadas" impidiendo realizar una transacción (con una calificación de 2.33 en ambos casos). Los datos arrojados para la dimensión de post venta, Amazon presenta un promedio del atributo de 3.73, encontrando que en los atributos RES2 (manejo de las devoluciones de productos), COM2 (compensación al consumidor cuando la orden no llega a tiempo) y COM3 (Recogida de las devoluciones en el domicilio del consumidor), se presentan las mayores distancias en relación a la máxima calificación posible.

Tabla 11. Resultados Promedio de las Dimensiones y Calificaciones Máximas Posibles de la Escala

\begin{tabular}{|c|c|c|c|c|c|c|c|}
\hline $\begin{array}{c}\text { Portal o } \\
\text { Empresa }\end{array}$ & Eficiencia & Disponibilidad & Privacidad & Cumplimiento & $\begin{array}{c}\text { Valor } \\
\text { Percibido }\end{array}$ & $\begin{array}{c}\text { Post } \\
\text { Venta }\end{array}$ & Lealtad \\
\hline Amazon & 4,39 & 3,46 & 4,08 & 4,23 & 5,65 & 3,73 & $\mathbf{4 , 0 8}$ \\
\hline $\begin{array}{c}\text { Calificación } \\
\text { Máxima } \\
\text { Posible }\end{array}$ & 5,00 & 5,00 & 5,00 & 5,00 & 7,00 & 5,00 & 5,00 \\
\hline
\end{tabular}

Fuente: elaboración propia

Figura 8. Promedio de las Dimensiones y Calificación Máxima Posible

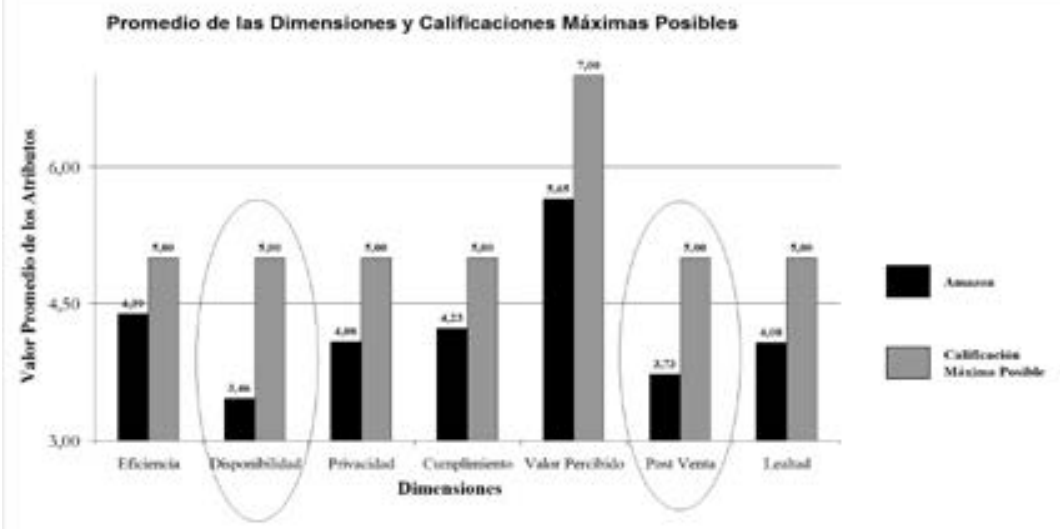

Fuente: Elaboración Propia 


\section{DISCUSIÓN Y CONCLUSIONES}

Mediante el presente estudio se ha ampliado la experiencia y conocimiento sobre la importancia de la calidad percibida en entornos de compra virtuales.

El estudio ha permitido aplicar las escalas e-SQ (E-S-QUAL y E-Rec-QUAL), a uno de los retailers más importantes del mundo en el contexto latinoamericano obteniendo una cantidad significativa de respuestas correctas que nos permitieron realizar el análisis correspondiente.

En las dimensiones de percepción de calidad de servicio (medidas por las escalas E-S-Q), Amazon presentó distancias significativas en relación a las calificaciones máximas posibles presentes en las escalas E-S-Q, en lo que respecta a disponibilidad del sistema y post venta. Específicamente, en lo que respecta a las dimensiones de calidad de servicio y en la dimensión de recuperación presenta brechas significativas en los atributos de post venta referidos al manejo de las devoluciones de productos, la compensación ante los problemas que crea, compensación cuando el pedido no llega a tiempo y la recolección de las devoluciones en el domicilio de los clientes.

En conclusión, los autores detectan grandes desafíos para la empresa en relación con la necesidad de ejecutar mejoras significativas en los aspectos de disponibilidad del sistema y de servicios post venta.

Los resultados también abren la oportunidad de aplicar el instrumento a nuevas empresas de distintas industrias y zonas geográficas con el objeto de verificar hasta qué punto es aplicable de manera general para determinar calidad de servicio percibida en negocios on line. 


\section{REFERENCIAS}

AHMAD, A., RAHMAN, O. Y KHAN, M. (2017) Exploring the role of website quality and hedonism in the formation of e-satisfaction and e-loyalty: Evidence from internet users in India Journal of Research in Interactive Marketing, vol. 11 No. 3, págs. 246-267.

BAKOS, JY., (1997). Reducing buyer search costs: Implications for electronic marketplaces.

BARNES, S.J, \& VIDGEN, R (2000). Information and interaction quality: Evaluating Internet bookshop Web site with Webqual. Proceedings of the 13th International Bled Electronic Commerce Conference, 426-444.

BAUER, H. H.; FALK, T.: HAMMERSCHMIDT, M. (2006). "eTransQual: A transaction process-based approach for capturing service quality in online shopping". Journal of Business Research, vol. 59, núm. 7, pp. 866875.

BOSHOFF, C. (2007). A PSYCHOMETRIC ASSESSMENT OF E-S-QUAL: A SCALE TO MEASURE ELECTRONIC SERVICE QUALITY. Journal of Electronic Commerce Research, 8(1), 101-114.

CARLSON, J. \& O'CASS, A. (2010), Exploring the relationships between e-service quality, satisfaction, attitudes and behaviours in content-driven e-service web sites, Journal of Services Marketing, vol. 24 No. 2, págs. 112-127.

CHEN, Q., RODGERS, S. \& HE, Y. (2008). A Critical Review of the E-Satisfaction Literature. American Behavioral Scientist, 52 (1), 38-59.

CONNOLLY, R. (2007): "Factors Influencing Irish Consumers iTrust in Internet Shopping", Management Research News: Communication of Emergent International Management Research, vol. 31, Issue 5.

COX, J. \& DALE, B. (2001). Service quality and e-commerce: An exploratory analysis. Managing Service Quality, 11 (2), 121-132.

CROSS, R. \& SMITH, J. (1995). Customer-focused strategies and tactics: interactive marketing weighs in for customers. In E. Forrest \& R. Mizerski (Eds.): Interactive Marketing: The future present. (pp. 5-28). NTC Publishing Group

DUQUE, E. J. (2005). Revisión del concepto de calidad del servicio y sus modelos de medición. Innovar. Revista de Ciencias Administrativas y Sociales, 25, 64-80. 
FASSNACHT, M. \& KOESE, I. (2006). Quality of electronic services: Conceptualizing and testing a hierarchical model. Journal of Service Research, 9 (1), 19-38.

GHOSH, M. (2018). Measuring electronic service quality in india using E-SQUAL. The International Journal of Quality \& Reliability Management, 35(2), 430-445.

\section{SPIROS GOUNARIS SERGIOS DIMITRIADIS VLASIS STATHAKOPOULOS,} (2010),"An examination of the effects of service quality and satisfaction on customers' behavioral intentions in e\#shopping", Journal of Services Marketing, Vol. 24 Iss 2 pp. $142-156$.

GRÖNROOS, CHRISTIAN (1982), Strategic Management and Marketing in the Service Sector. Helsingfors, Sweden: Swedish School of Economics and Business Administration.

GWO-GUANG, LEE \& HSIU-FEN, LIN. (2005). Consumer perceptions of e-Service quality in online shopping. International Journal of Retail \& Distribution Management. 33. 161-176.

KIM SY, LIM YJ. Consumers Perceived Importance of and Satisfaction with Internet Shopping. Electronic Markets 2001; 11(3): 148-154.

LADHARI, R. (2010). Developing e-service quality scales: A literature review. Journal of Retailing and Consumer Services, 17 (6), 464-477.

LAUDON, C. K. \& TRAVER, C. G. (2014). E-Commerce. Business. Technology. Society. Published by Pearson Education, Inc., One Lake Street, Upper Saddle River, New Jersey 07458.

LEHTINEN, UOLEVI AND JARMO R. LEHTINEN (1982), "Service Quality: A Study of Quality Dimensions," unpublished working paper, Service Management Institute, Helsinki, Finland.

LIM H, DUBINSKY AJ. Consumers' perceptions of e-shopping characteristics: an expectancy-value approach", Journal of Services Marketing 2004; 18(7): 500-513.

MALLOR, ELODIE. (2017). L'évolution dans le temps du poids des dimensions de l'expérience sur la satisfaction cumulée: le cas des services hédoniques.

MICK, DAVID GLENN AND SUSAN FOURNIER (1995), "Technological Consumer Products in Everyday Life: Ownership, Meaning, and Satisfaction," working paper, report No. 95-104, 1-59, Marketing Science Institute, Cambridge, MA —and - (1998), "Paradoxes of Technology: Consumer Cognizance, Emotions, and Coping Strategies," Journal of Consumer Research, 25 (September), 123-47. 
MODAES LATINOAMERICA (26 de septiembre de 2018). Amazon da forma a su entrada en Chile: se instalará en una bodega en Santiago. https:// www.modaes.com/empresa/amazon-da-forma-a-su-entrada-en-chilese-instalara-en-una-bodega-de-santiago.html

MURPHY, J., HOFACKER, C.F., \& BENNETT, M. (2001). Website-generate market-research data. Hotel and Restaurant Administration Quarterly, 42 (1), 82-91.

PARASURAMAN, A. \& ZINKHAN, G. M. (2006). Marketing to and serving customers through the Internet: An overview and research agenda. Journal of the Academy of Marketing Science, 30 (4), 286-295.

PARASURAMAN, A., ZEITHAML, V. \& BERRY, L. (1988). SERVQUAL: A multipleitem scale for measuring consumer perceptions of service quality. Journal of Retailing, 64 (1), 12-40.

PARK, C.; KIM, Y. (2003). "Identifying key factors affecting consumer purchase behavior in an on-line shopping context". International Journal of Retail \& Distribution Management, vol. 31, núm. 1, pp.16-29.

PENTINA, IRYNA \& AMIALCHUK, ALIAKSANDR \& TAYLOR, DAVID. (2011). Exploring effects of online shopping experiences on browser satisfaction and e-tail performance. International Journal of Retail \& Distribution Management. 39. 742-758. 10.1108/09590551111162248.

RAFIQ M.; LUX. AND FULFORD H. (2011). "Measuring Internet retail service quality using E-S-QUAL”, Journal of Marketing Management, vol. 27. Issue. 9-10. pp. 1-15.

ROWLEY, J. (2006). An analysis of the e-service literature: Towards a research agenda. Internet Research, 16 (3), 339-359.

SANTOURIDIS I., TRIVELLAS P., TSIMONIS G. (2012) "Using E-S-QUAL to measure internet service quality of e-commerce web sites in Greece", International Journal of Quality and Service Sciences, vol. 4. Iss: 1, pp.8698.

SASSER, W. EARL, JR., R. PAUL OLSEN, AND D. DARYL WYCKOFF (1978), Management of Service Operations: Text and Cases. Boston: Allyn \& Bacon.

SARKAR, R., \& DAS, S. (2016). E-retailing: Boon or Bane? International Journal of Management Research and Reviews, 6(1), 9.

SCHLAUCH ANDREW \& STEVEN LAPOSA (2001) E-tailing and Internet-related real estate cost savings: A comparative analysis of E-tailers and retailers. Journal of Real Estate Research: 2001, vol. 21, núm. 1-2, págs. 43-54. 
SHANKAR, AMIT \& DATTA, BIPLAB. (2020). Measuring e-service quality: a review of literature. International Journal of Services Technology and Management. 26. 77. 10.1504/IJSTM.2020.105398.

SETH, N., DESHMUKH, S. G. \& VRAT, P. (2005). Service quality models: a review. International Journal of Quality \& Reliability Management, 22 (9), 913-949.

WANG, Y. S., \& TANG, T. I. (2003). Assessing customer perceptions of website service quality in digital marketing environments. Journal of End User Computing, 15(3); 14-31.

WATSON, R.T., AKSELSEN, S., AND PITT, L.F. "Attractors: building mountains in the flat landscape of the World Wide Web," California Management Review (40:2) 1998, pp 36-56

WOLFINBARGER, M., \& GILLY, M.C. (2003). Etailq: Dimensionalizing, measuring and predicting etail quality. Journal of Retailing, 79, 183-198.

WU, XIN \& LIU, FEIYAN. (2018). An Analysis of the Motivation of Customer Participation Value Co-Creation in the We-Media: A Study Based on Content Marketing. Open Journal of Business and Management. 06.

YANG, H.; TSAI, F. (2007). "General E-S-QUAL Scales Applied To Websites Satisfaction and Loyalty Model". Communications of the IIMA 117, vol. 7, núm. 2, pp. 115-126.

YAYA L. H. P., MARIMON F., CASADESUS M. (2011). "Customer's loyalty and perception of ISO 9001 in online banking", Industrial Management \& Data Systems, vol. 111 Iss: 8, pp. 1194-1213.

PETNJI YAYA, L. H., MARIMON, F., \& CASADESÚS, M. (2017). The expert experience in adopting the E-S-QUAL scale. Total Quality Management \& Business Excellence, 28(11-12), 1307-1321.

YOO, BOONGHEE \& DONTHU, NAVEEN. (2001). Developing and Validating a Multidimensional Consumer-Based Brand Equity Scale. Journal of Business Research.

ZEITHAML, V., PARASURAMAN, A. \& MALHOTRA, A. (2000). A conceptual framework for understanding e-service quality: Implications for future research and managerial practice. [Working Paper № 100- 115/ WEP 1-11]. Marketing Science Institute. Cambridge. 


\section{ANEXO: ENCUESTA}

EFF1 Este sitio hace que sea fácil encontrar lo que necesito. *

$\bigcirc$ Siempre

$\bigcirc$ Casi Siempre

$\bigcirc$ A veces

Casi Nunca

Nunca

EFF2 Hace que sea fácil llegar a cualquier parte del sitio. *

Siempre

Casi Siempre

$\bigcirc$ A veces

$\bigcirc$ Casi Nunca

Nunca

EFF3 Me permite completar una transacción rápidamente. *

Siempre

$\bigcirc$ Casi Siempre

$\bigcirc$ A veces

Casi Nunca

Nunca

EFF4 La información en este sitio está bien organizada. *

Siempre

$\bigcirc$ Casi Siempre

$\bigcirc$ A veces

Casi Nunca

Nunca 
EFF5 Carga sus páginas rápido. *

$\bigcirc$ Siempre

$\bigcirc$ Casi Siempre

$\bigcirc$ A veces

$\bigcirc$ Casi Nunca

Nunca

EFF6 Este sitio es fácil de usar. *

$\bigcirc$ Siempre

$\bigcirc$ Casi Siempre

$\bigcirc$ A Veces

Casi Nunca

Nunca

EFF7 Este sitio me permite acceder a él rápidamente. *

$\bigcirc$ Siempre

$\bigcirc$ Casi Siempre

$\bigcirc \quad$ A Veces

Casi Nunca

$\bigcirc$ Nunca

EFF8 Este sitio está bien organizado. *

$\bigcirc$ Siempre

$\bigcirc$ Casi Siempre

A Veces

$\bigcirc$ Casi Nunca

$\bigcirc$ Nunca 
SYS1 Este sitio siempre está disponible para Operar. *

$\bigcirc$ Siempre

$\bigcirc$ Casi Siempre

A Veces

$\bigcirc$ Casi Nunca

Nunca

SYS2 Este sitio se inicia y se ejecuta de inmediato. *

$\bigcirc$ Siempre

Casi Siempre

$\bigcirc$ A Veces

Casi Nunca

Nunca

SYS3 Este sitio se bloquea. *

Siempre

Casi Siempre

$\bigcirc$ A Veces

Casi Nunca

$\bigcirc$ Nunca

SYS4 Las páginas de este sitio se quedan "pegadas o congeladas" después de ingresar a mi información del pedido. *

Siempre

Casi Siempre

- A Veces

$\bigcirc$ Casi Nunca

$\bigcirc$ Nunca 
PRI1 Protege la información sobre mi comportamiento de compra en la web. *

$\bigcirc$ Siempre

$\bigcirc$ Casi Siempre

$\bigcirc$ A Veces

Casi Nunca

Nunca

PRI2 Este sitio comparte mi información personal con otros sitios. *

Siempre

Casi Siempre

$\bigcirc$ A Veces

$\bigcirc$ Casi Nunca

Nunca

PRI3 Este sitio protege información sobre mi tarjeta de crédito o medio de pago. *

$\bigcirc$ Siempre

Casi Siempre

$\bigcirc$ A Veces

Casi Nunca

$\bigcirc$ Nunca

FUL1 Entrega las órdenes cuando se prometió. *

- Siempre

Casi Siempre

A V Veces

$\bigcirc$ Casi Nunca

Nunca 
FUL2 Este sitio hace que los artículos estén disponibles para su entrega dentro de un marco de tiempo adecuado. *
Siempre
Casi Siempre
A Veces
Casi Nunca
Nunca

FUL3 Entrega rápidamente lo que ordeno. *
Siempre
Casi Siempre
$\bigcirc$ A Veces
Casi Nunca
Nunca

FUL4 Envía los artículos ordenados. *

Siempre

Casi Siempre

A Veces

$\bigcirc$ Casi Nunca

Nunca

FUL5 tiene en stock los artículos que la compañía afirma tener. *
Siempre
Casi Siempre
A Veces
$\bigcirc$ Casi Nunca
Nunca 
FUL6 Es veraz sobre sus ofertas. *

$\bigcirc$ Siempre

Casi Siempre

$\bigcirc$ A Veces

Casi Nunca

Nunca

FUL? Hace promesas precisas sobre la entrega de productos. *

Siempre

$\bigcirc$ Casi Siempre

A Veces

Casi Nunca

Nunca

RES1 Me proporciona opciones convenientes para devolver artículos. *

$\bigcirc$ Siempre

$\bigcirc$ Casi Siempre

$\bigcirc$ A Veces

$\bigcirc$ Casi Nunca

$\bigcirc$ Nunca

RES2 Este sitio maneja bien las devoluciones de productos. *

$\bigcirc$ Siempre

Casi Siempre

$\bigcirc$ A Veces

Casi Nunca

Nunca 
RES3 Este sitio ofrece una garantía significativa. *

$\bigcirc$ Siempre

$\bigcirc$ Casi Siempre

$\bigcirc$ A Veces

Casi Nunca

Nunca

RES4 Me dice qué hacer si mi transacción no es procesada. *

$\bigcirc$ Siempre

Casi Siempre

$\bigcirc$ A Veces

Casi Nunca

Nunca

RES5 Se ocupa de los problemas a la brevedad. *

Siempre

Casi Siempre

$\bigcirc$ A Veces

Casi Nunca

$\bigcirc$ Nunca

C0M1 Este sitio me compensa por los problemas que crea. *

$\bigcirc$ Siempre

Casi Siempre

$\bigcirc$ A Veces

$\bigcirc$ Casi Nunca

$\bigcirc$ Nunca 
COM2 Me compensa cuando lo que ordené no llega a tiempo. *

$\bigcirc$ Siempre

$\bigcirc$ Casi Siempre

A Veces

$\bigcirc$ Casi Nunca

$\bigcirc$ Nunca

COM3 Recoge los articulos que quiero devolver de mi casa o negocio. *

Siempre

Casi Siempre

$\bigcirc$ A Veces

Casi Nunca

Nunca

CONI Este sitio proporciona un número de teléfono para acceder a la empresa. *

Siempre

Casi Siempre

$\bigcirc$ A Veces

Casi Nunca

Nunca

CON2 Este sitio tiene representantes de servicio al cliente disponibles en linea. *

$\bigcirc$ Siempre

$\bigcirc$ Casi Siempre

A V Veces

$\bigcirc$ Casi Nunca

$\bigcirc$ Nunca 
CON3 Ofrece la posibilidad de hablar con una persona viva (no un sistema automatizado), si existe un problema.
Siempre
Casi Siempre
A Veces
Casi Nunca
Nunca

\section{Califique al Sitio Web Utilizando una Escala de 1 a 7 , siendo 1 deficiente y 7 excelente}

VPE1 Los precios de los productos y servicios disponibles en el sitio. *

$\begin{array}{rrrrrrl}1 & 2 & 3 & 4 & 5 & 6 & 7 \\ \text { Deficiente } \bigcirc & \bigcirc & \bigcirc & \bigcirc & \bigcirc & \bigcirc & \bigcirc \text { Excelente }\end{array}$

VPE2 La conveniencia general de usar este sitio. *

$\begin{array}{rrrrrrr}1 & 2 & 3 & 4 & 5 & 6 & 7 \\ \text { Deficiente } \bigcirc & \bigcirc & \bigcirc & \bigcirc & \bigcirc & \bigcirc & \bigcirc \text { Excelente }\end{array}$

VPE3 La medida en que el sitio te da una sensación de tener el control. *
1
23
4
5
$6 \quad 7$
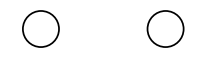
$\bigcirc$

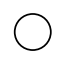
$\bigcirc$ Excelente

VPE4 El valor total que obtiene de este sitio por su dinero y esfuerzo. *

$\begin{array}{rrrrrrc}1 & 2 & 3 & 4 & 5 & 6 & 7 \\ \text { Deficiente } \bigcirc & \bigcirc & \bigcirc & \bigcirc & \bigcirc & \bigcirc & \bigcirc \text { Excelente }\end{array}$




\section{Califique al Sitio Web Utilizando una Escala de 1 a 5, siendo 1 muy improbable y 5 muy probable.}

LEA1 ¿Qué tan probable es que usted diga cosas positivas sobre este sitio a otras personas?

$\begin{array}{lllllll} & 1 & 2 & 3 & 4 & 5 & \\ \text { Muy Improbable } & \bigcirc & \bigcirc & \bigcirc & \bigcirc & \bigcirc & \text { Muy Probable }\end{array}$

LEA2 ¿Qué tan probable es que usted recomiende este sitio a alguien que busca tu consejo?

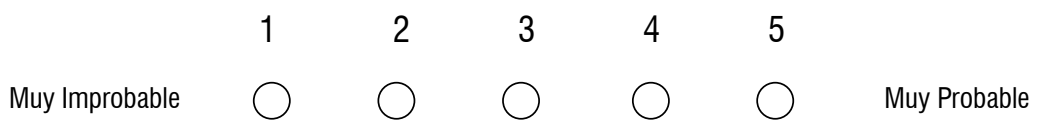

LEA3 ¿Qué tan probable es que usted anime a amigos y otras personas a hacer compras en este sitio?
Muy Improbable
2
3
4
5

$\begin{array}{lrrrrr}1 & 2 & 3 & 4 & 5 & \\ & \bigcirc & \bigcirc & \bigcirc & \bigcirc & \text { Muy Probable }\end{array}$

LEA4 ¿Qué tan probable es que usted considere a este sitio como su primera opción para futuras transacciones? *
Muy Improbable
23
4
5

$\begin{array}{lllll}1 & 2 & 3 & 4 & 5\end{array}$

LEA5 ¿Qué tan probable es que usted haga más compras en este sitio en los próximos meses?*

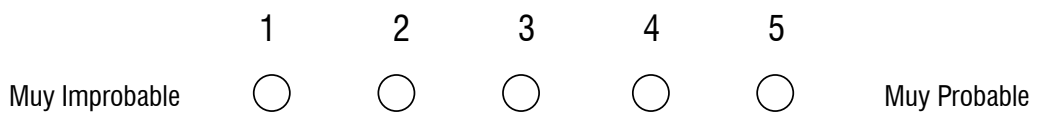

\section{Datos de quien Contesta}

DDE1 Sexo de quien responde*
Femenino
Masculino 
DDE2 Edad de quien responde *

DDE3 Cuál forma de pago utilíza mayoritariamente para el pago de sus compras?*

Tarjeta de Débito/ Cuenta Rut

Tarjeta de Crédito Bancaria

Tarjeta de Crédito del Supermercado

Tarjeta de Crédito de Casa Comercial

PayPal

$\bigcirc$ Otro medio de pago

DDE4 Región de residencia *

DDE5 Comuna de residencia*

DDE6 Nivel Educacional de quien responde: *

Básica incompleta

Básica completa

Media incompleta

$\bigcirc$ Media Completa

Técnico Profesional incompleta

Técnico Profesional completa

Universitaria incompleta

Universitaria completa

Post grado incompleto

Post grado completo

DDE7 Número de componentes del hogar *

DDES Su nivel de ingreso familiar se encuentra en el siguiente rango: * 